\title{
Biomechanical changes on the typical sites of pressure ulcers in the process of turning over from supine position: theoretical analysis, simulation and experiment
}

\section{Peng Su ( $\sim$ supeng@bistu.edu.cn )}

Beijing Information Science and Technology University - Qinghe Xiaoying Campus: Beijing Information Science and Technology University

\section{Qinglong Lun}

Beijing Information Science and Technology University

\section{Da Lu}

BeiHang University School of Biological Science and Medical Engineering

\section{Qiulong Wu}

Beijing Information Science and Technology University

Tian Liu

Beijing Information Science and Technology University

\section{Leiyu Zhang}

Beijing University of Technology

\section{Research Article}

Keywords: turning over, pressure ulcers, contact pressure, biomechanical simulation, rehabilitation technical aids

Posted Date: August 2nd, 2021

DOI: https://doi.org/10.21203/rs.3.rs-772442/v1

License: (c) (i) This work is licensed under a Creative Commons Attribution 4.0 International License. Read Full License

Version of Record: A version of this preprint was published at Annals of Biomedical Engineering on April 8th, 2022. See the published version at https://doi.org/10.1007/s10439-022-02938-9. 


\section{Biomechanical changes on the typical sites of pressure ulcers}

\section{2 in the process of turning over from supine position:}

\section{3 theoretical analysis, simulation and experiment}

4 Peng $\mathrm{Su}^{1}$, Qinglong Lun ${ }^{1}$, $\mathrm{Da} \mathrm{Lu}{ }^{2}$, Qiulong Wu ${ }^{1}$, Tian Liu ${ }^{1}$,

5 Leiyu Zhang ${ }^{3 *}$

ABSTRACT

Background: Pressure ulcer is a typical disease, which is common in long-term bedridden patients and difficult to cure. It is necessary to study the biomechanics of the typical sites of pressure ulcers in turning over from supine position, which is an important reference for clinical medical nursing and and guides an assisted exoskeleton robot design.

Methods: The typical sites of pressure ulcers mainly focus on the scapula and the hip-sacrum of the trunk in turning over from the supine position. Based on the requirements of rehabilitation technical aids and the anatomy theory, the simple model of the scapula and the hip-sacrum were established for a force analysis in the process of turning over from the supine position, and the theoretical contact pressure between the human body and the bed surface was obtained. Then, three-dimensional models of the scapula and hip- sacrum were reconstructed and the maximum stress under different boundary conditions was obtained by finite element analysis. Finally, the pressure distribution sensor was used to carry out the human experiment of turning over from the supine position, and the pressure cloud diagram and the maximum contact pressure curve of the shoulder blade and the hip were obtained

\footnotetext{
* Correspondence information: zhangleiyu1988@ 126.com; ${ }^{3}$ Beijing Key Laboratory of Advanced Manufacturing Technology, Beijing University of Technology, No.100 Pingleyuan, Chaoyang District, Beijing100124, China. Full list of author information is available at the end of the article.
} 
under different angles of turning over.

Results: The results from theoretical analysis, simulation and experiment were almost the same change trends, and the curves and the stress diagrams showed the contact pressure change of the typical sites of pressure ulcers in turning over. The angle threshold of the optimal comprehensive pressure can improve the use efficiency of the equipment to assist human turning over and reduce the incidence of pressure ulcers in the use of assisted bed in long-term bedridden patients.

Conclusions: In response to the less research on the mechanism of pressure ulcer, biomechanical changes have been revealed, which helps to explain the causes of pressure ulcer disease and provide basis for improving clinical nursing, and the relevant results provided a reference that contributes to the man-machine coupling design of the assisted rollover robot.

Key word: turning over, pressure ulcers, contact pressure, biomechanical simulation, rehabilitation technical aids

\section{Background}

The pressure ulcer, also known as decubitus, is localized injury to the skin and/or subcutaneous tissue caused by pressure or pressure in combination with shearing force [1]. This phenomenon has a physiological effect restricting blood flow, with the restriction in blood flow, perfusion of oxygen is limited and removal of metabolic waste is inhibited which leads to cells death in the affected area resulting in ischemia followed by pressure ulcers formation [2]. Pressure ulcers usually occur in areas of bony prominence, for instance, the elbow, heel and back of skull, and Multiple mechanical parameters including friction, shear stress, pressure and microclimate are believed to be included in the potential triggering factors [3-6]. It is more cost-effective to prevent the occurrence of ulcers than provide treatment 
especially for the stage four ulcers [7]. The risk of death after suffering the pressure ulcers will increase 4-fold in the critical patients [8]. Nevertheless, the incidence of pressure ulcers can decrease by $50-60 \%$ through effective prevention [9]. So it is significant to study the biomechanics of the typical sites of pressure ulcers in turning over from supine position, and it helps to achieve better care.

The prevention of pressure ulcers is mainly about relieving backpressure, decrease the exposure time and improving skin health [10]. The common method is to change the patient's body position every two hours by turning over, and as technology advances, rehabilitation robots may also be used that needs to have good human-machine coupling [11-13]. Seo et al. discussed the development of an intelligent bed robot system, and monitoring using an array of pressure sensors, the bed posture and movement of the patients were measured and evaluated [14]. Some scholars model the bones and muscles of the human body movement system based on the skeletal structure, and other studies on how to prevent pressure ulcers are in-depth discussion [15]. Recently the new type sheet with low friction and optimized moisture transfer properties has been studied, which may be used to prevent pressure ulcers because of its lower friction properties compared with a common medical sheet [16-18]. Furthermore, the relationship between the contact pressure and the supine angle was studied by researchers. They conclude that the minimum and maximal contact pressures of the sacrum are in a supine angle of $30^{\circ}$ and $90^{\circ}[19]$.

Current research on pressure ulcer care mainly focuses on the pressure relief equipment such as the decompression mattress or rehabilitation robot. There are few investigations into the biomechanics of human structure leading to pressure ulcers. According to the structure of the body, the scapula, sacrum and calcaneus form a 
plane and support the entire body weight in the supine position [20], and the tilting can affect the strain distribution, taking away the highest peak strains of the sacrum. In different parts of the body, the probability of pressure ulcers is different, and in the same area, there are biomechanical changes in the process of turning over. It is necessary to reveal the biomechanics mechanism leading to pressure ulcers in order to better guide pressure ulcers care, such as the optimal care angle and the specific care position.

For the purpose of explaining the biomechanical mechanism of human turning over movement from supine position, the paper explores the interaction between the typical sites of pressure ulcers and stress condition in the process of turning over from supine position, then the result of the experiment in which the body side turn $90^{\circ}$ can be observed. After that, this paper implements three-dimensional reconstruction of the human skeleton with the help of CT (Computed tomography) scan image and finite element analysis by ABAQUS to analyze the stressful condition of human body. Finally, the contact pressures between the common sites of pressure ulcers and the sensor are obtained by means of the pressure distribution test sensor. Comparing the calculation results, simulation results and experimental results, the variation in pressure of the body in the supine position is obtained.

\section{Methods}

From an anatomical perspective, the pressure ulcers are prone to occur in skeletal protuberance in which the place under pressure and lack of fat protection, no muscle wrap or muscle thin. Without the muscle between the bone and the skin to bear the buffer and decompression, the stress concentration phenomenon will occur in the skin of skeletal protuberance when the skeletal protuberance bears the vertical pressure. The following image shows the typical sites of pressure ulcers in different positions of 
the human body in the bed (Fig. 1), where the position of pressure ulcers on only one side is shown. The red circles represent the location of pressure ulcers and the area of pressure ulcers that may occur. Because most of the body weight concentrates in the upper body and the stress on one side of the body increases in turning over from the supine position, the typical and main sites of pressure ulcers is focused on the scapula and hip-sacrum in this movement.

\section{Theoretical force analysis of the main pressure ulcer sites}

The skeletal protuberance of the shoulder and hip are the main site of pressure ulcers in the process of turning over from the supine position. Furthermore, the vertical force related to shear force is the main factor in the occurrence of pressure ulcers. Therefore, the study on the contact pressure between the skeletal protuberance of the shoulder and hip and the horizontal bed surface can better explain the cause and prevention of pressure ulcers in a different angle of the supine position.

The MIMICS (Materialise's interactive medical image control system) software is used to carry out three-dimensional reconstruction of the human skeleton, and the models of the scapula and hip-sacrum were obtained [21,22]. Then, through theoretical analysis and calculation of bone extrusion soft tissue in the process of turning over from the supine position, the contact pressure changes between the main sites of pressure ulcers and the horizontal bed surface will hopefully be obtained. Because the right direction of turning over from the supine position is symmetric with the left direction, the rightward roll motion was analyzed only. The extrusion force on one side of the body increases in turning over from the supine position. Theoretical stress analysis of the scapula in the process of turning over from supine position is shown in Fig. 2. When the angle of the lateral position 
is $\theta$, the uniformly distributed load is

$$
F_{N 1}=G_{R s b}+\left(G_{B}+G_{L s b}\right)(1-\cos \theta)
$$

Where the scapular and the spine initial stress are $G_{R s b}$ and $G_{B}$. The angle between the scapular plane and the chest plane is $30^{\circ}$ [23]. Among them, the stress at scapula is respectively $F_{N 1}$ and $F_{N 2}$. The gravity around the spine is $G_{B}$.

In addition to the scapula, the hip-sacrum is also prone to pressure ulcers in turning over from the supine position. The sacrum and tailbone protrude outward from the hip-sacrum in the supine position of the body. Thus, the sacrum and tailbone bear the main body pressure. If the vertical stress of the sacrum and tailbone can be changed to the lateral stress, the incidence of the pressure ulcers will be reduced greatly. The theoretical force analysis of the hip-sacrum in the process of turning over from supine position is shown in Fig. 3. Uniformly distributed load is

$$
F_{N 4}=\lambda G_{H}+(1-\lambda)(1-\cos \theta) G_{H}
$$

Where the gravity at hip-sacrum is $G_{H} . \lambda$ is the ratio of the initial weight of one hip to the total weight of the hip $G_{H}$. Among them, the stress at sacrum is $F_{N 3}$. The stress at hip is $F_{N 4}$. Based on medical software measurement, the angle between the hip plane and the chest plane is about $45^{\circ}$.

When the sacrum does not touch the bed, $F_{N 3}=0$.

\section{Biomechanical modeling of the main pressure ulcer sites}

Based on the CT scan slices of a healthy man, the three-dimensional reconstruction of the bone was carried out [24]. Then, the three-dimensional bone was imported to the Geomagic Studio software for optimization. Finally, the optimized bone was imported to the ABAQUS for biomechanical modeling [15, 24].

The bone is divided into the cortical bone, cancellous bone, articular cartilage and so on, and the material properties of the different parts of the bone are not the same. 
At present, most studies have simplified the bone into the cortical bone and cancellous bone, and regarded them as homogeneous and isotropic linear elastomers [25]. To simplify the study, the cortical bone and cancellous bone are regarded as the same material properties [26-28]. The Elasticity of the scapula is $9 \mathrm{GPa}$, Poisson's ratio is 0.3 [29]. The Elasticity of the Pelvis is $43.53 \mathrm{GPa}$ and the Poisson's ratio is 0.2 [30].

Stress is generated when bones crush soft tissue and stress concentrations lead to pressure ulcers. Although soft tissue includes muscle, skin and so on, in order to improve the efficiency of the simulation, the soft tissue is viewed as a whole and its Elasticity is $200 \mathrm{kPa}$ and the Poisson's ratio is 0.459 [31].

Different boundary conditions will be applied to the scapula and hip-sacrum in different states based on the theoretical force analysis of Fig. 2 and Fig. 3. The boundary conditions of five angles of $0^{\circ}, 30^{\circ}, 45^{\circ}, 60^{\circ}$ and $90^{\circ}$ were set.

The details of the model of the scapula and hip-sacrum are used to illustrate the principal constraint setting better, as shown in Fig. 4. In this figure, $\varphi$ is the angle between the force line and the plane of the shoulder blade, $\mathrm{F}$ is the vector force applied to the bone, and represents the direction of the force from the inside out. The force on the left side is always straight down and the angle between the scapula and the horizontal plane changes. The force on the right side always points the horizontal plane from the inside of the hip. Based on the above analysis, principal constraint settings are showed that includes boundary conditions and loads, as shown in Table. 1. For the model details in the Fig. 4, constraints are set to try to keep up with reality. 
Tabel 1. Principal constraint settings between the soft tissue and bone

\begin{tabular}{cccc}
\hline Angle & Constraint & Scapula & Hip-sacrum \\
\hline \multirow{2}{*}{$0^{\circ}$} & Boundary Conditions & Tie with soft tissue, (3)(4) & Tie with soft tissue, (8)(11) \\
& Load & $\varphi=60^{\circ},(5)$ & $F=F \cos 45^{\circ}$, (12) \\
$30^{\circ}$ & Boundary Conditions & Tie with soft tissue, (3)(4)(7) & Tie with soft tissue, (8)(9)(11) \\
& Load & $\varphi=90^{\circ},(5)$ & $F=F \cos 75^{\circ}$, (12) \\
$45^{\circ}$ & Boundary Conditions & Tie with soft tissue, (2)(3)(4) (7) & Tie with soft tissue, (8)(9)(11) \\
& Load & $\varphi=105^{\circ}$, (5) & $F=F \cos 90^{\circ}$, (12) \\
$60^{\circ}$ & Boundary Conditions & Tie with soft tissue, (2)(3)(4)(7)(1) & Tie with soft tissue, (8)(10)(11) \\
& Load & $\varphi=120^{\circ}$, (5) & $F=F \cos 105^{\circ}$, (12) \\
$90^{\circ}$ & Boundary Conditions & Tie with soft tissue, (2)(3)(4)(7)(1) & Tie with soft tissue, (8)(11)(13) \\
& Load & $\varphi=150^{\circ}$, (5) & $F=F \cos 135^{\circ}$, (12) \\
\hline
\end{tabular}

\section{Contact pressure measurement experiment in the process of turning over}

179 In order to explain the laws of motion, and to verify the correctness of the theoretical analysis and biomechanical modeling, the contact pressure between the shoulder blade and hip and the bed surface in the process of turning over need to be measured experimentally. The measurement subject is a healthy volunteer, male, 24 years old, $175 \mathrm{~cm}$ in height, $70 \mathrm{~kg}$ in weight, with no adverse health risks, meeting the requirements of the experiment. Before the experiment, the subject had filled in the informed consent form and completed the relevant review. contains a grid of semiconductor substrates, which can change the resistance value of the internal elements after the load pressure. And the most basic sensor is constituted by these interlaced matrixes. The resistance data of each sensing element can be measured through rapid electronic scanning, and the size, time and position of the

194 force and pressure on the sensor can be obtained through simple calibration function. The pressure distribution test sensor can complete the real-time acquisition, display 
and storage of multi-channel sensor information, and can realize the calculation and analysis functions such as the contact image description, the pressure map, contact area and pressure center trajectory of various parts of the human body.

Contact pressure measurement experiment in the process of turning over will be performed as shown in Fig. 5. To begin with the subject is lying flat on the pressure sensor, and the shoulder blade is in contact with the sensor. The subject does rollover in an independent supine position, and the pressure change data of the human shoulder blade is collected. Then the subject is lying flat on the pressure sensor, and the hip is in contact with the sensor. The subject does the supine roll with an independent supine position, and the pressure change data of the human hip is collected.

\section{Results}

\section{Theoretical force analysis results}

According to the weight parameters of the human body, the following assumptions are made: $G_{B}$ is $150 \mathrm{~N}, G_{R \mathrm{sb}}$ and $G_{L \mathrm{sb}}$ are $30 \mathrm{~N}, G_{H}$ is $400 \mathrm{~N}$. According to human anatomy, the ratio of shoulder blade width at the upper arm to that at the back is about 2:3. The ratio $\lambda$ of the initial weight of one hip to the total weight of the hip $G_{H}$ is 0.5 .

Considering the contact stress and contact area between the scapula and hip-sacrum and the bed surface are varied, the stress should be used as the standard to evaluate the best lateral position. The length information of the contact area between the scapula and hip-sacrum and bed surface was measured by MIMICS. And then, the area of the contact area was calculated by CAD (Computer Aided Design) and the area in different states was estimated according to the shape of the bone. Finally, the 
pressure changes of the scapula and hip-sacrum were calculated using the pressure equation $P=F / S$.

The theoretical contact pressure of the scapula and hip-sacrum was obtained, as shown in Fig. 6. In this figure, the pressure of the scapula fell slightly within the range $0^{\circ}$ to $20^{\circ}$, rose a small within the range $20^{\circ}$ to $64^{\circ}$ and then rose significantly within the range $64^{\circ}$ to $90^{\circ}$. And the pressure of hip-sacrum fell largely within the range $0^{\circ}$ to $50^{\circ}$ and rose dramatically within the range $50^{\circ}$ to $90^{\circ}$. And the lowest pressure value of the scapula and the hip-sacrum occurred in the $20^{\circ}$ and $50^{\circ}$ respectively. The $\mathrm{d} 1$ is about $1.5 \mathrm{e} 4 \mathrm{~Pa}$ and $\mathrm{d} 2$ is about $1.56 \mathrm{e} 5 \mathrm{~Pa}$.

\section{Finite element analysis results}

Based on biomechanical modeling of the main pressure ulcer sites, the finite element simulation of the shoulder and hip is executed, and the stress changes were obtained as shown in Fig. 7.

In the stress diagram of the scapula and nearby soft tissue, the upper and lower angles of the scapula, the medial margin, and part of the scapulae are in contact with the nearby soft tissue. And the stress region are mainly occurred in the Area I. Then with the angle of the body in lateral position increased, most of the region of the scapular ridge touches the nearby soft tissue, and the stress changes area gradually concentrates on the Area II. The area of stress concentration achieves a shift from Area I to Area II during the stress change. At a lateral rotation angle of about 30 degrees, the contact area between the scapula and nearby soft tissues is relatively large, and the stress is more evenly distributed over the soft tissues. Lastly, when the lateral position is $90^{\circ}$, stress changes are mainly concentrated on the Area II.

In the stress diagram of the hip-sacrum and nearby soft tissue, the tailbone and sacrum are in contact with the nearby soft tissue in the supine position of $0^{\circ}$. And with 
the angle of the body in lateral position increased, the main stress gradually shifted from Area III to the Area IV and the contact area between the iliac crest and the bed surface is the largest in the lateral position about $45^{\circ}$. The stress difference of the scapula nearby soft tissue $\mathrm{d} 1$ is about $6.8 \mathrm{e} 3 \mathrm{~Pa}$ between the $0^{\circ}$ and $30^{\circ}$ and the stress difference of the hip-sacrum nearby soft tissue $\mathrm{d} 2$ is about $5.98 \mathrm{e} 4 \mathrm{~Pa}$ between the $0^{\circ}$ and $45^{\circ}$.

The figure (c) shows that the stress of the scapula decreased slightly within the range $0^{\circ}$ to $30^{\circ}$ and then rose significantly within the range $30^{\circ}$ to $90^{\circ}$. Moreover, the stress of the hip-sacrum decreased in the $0^{\circ}$ to $45^{\circ}$ and increased in the $45^{\circ}$ to $90^{\circ}$. And the lowest pressure value of the scapula and the hip-sacrum occurred about the $30^{\circ}$ and $45^{\circ}$ respectively.

\section{Experiment results}

The pressure distribution of the shoulder blade and hip was obtained after the experiment, as shown in Fig. 8. The horizontal and vertical lines are used to establish absolute coordinates and the position of the shoulder blades can be got. The geometric center of the shoulder blades is gradually moving to the left sides and the maximum pressure area is gradually moving up to the left. And the geometric center of the hip is gradually moving down to the left sides and the same as the maximum pressure area.

In the figure (a), the region (1) and (2) is the pressure region on both sides of the scapula, and the pressure at the scapula is the largest in the supine position. The region (3) in figure (b) is the largest pressure point, and at this moment, the left side of the body gradually disengages from the pressure sensor, and the center of gravity shifts to the right side of the body. Figure(c) shows that the contact area between the human body and the sensor is further shifted to the right side of the body. The largest 
pressure point in the figure (d) is the region (6) and the mean pressure in the lumbar goes down. This is consistent with the results of the previous theoretical calculation, and verifies the correctness of the theoretical analysis.

As shown in figure (e), the region (7) is the sacrum and the largest pressure region is in the sacrum. Figure (f) shows that the maximum pressure region is transferred from the sacrum region to the hip region, and the region (8) moves down to the right of the human body relative to the region (7). The pressure is distributed in most areas of the hip bone, and the contact area is the maximum. Figure (g) shows that the maximum pressure region gradually transferred from the hip region to the greater trochanter, and the region (9) continued to move to the lower right of the human body relative to the region (8), and the pressure distribution gradually concentrated in the region (9). Figure (h) shows that the largest pressure region on concentrate in the region (10), and the contact area decrease. The pressure distribution shows the characteristics of concentration to the dispersion to concentration with the angel increased in turning over from supine position.

This figure (i) shows that the stress of the regio scapularis gradually rose within the range $0^{\circ}$ to $25^{\circ}$, relatively stable within the range $25^{\circ}$ to $40^{\circ}$ and rose significantly within the range $40^{\circ}$ to $84^{\circ}$. The stress of hip-sacrum gradually fell in the $0^{\circ}$ to $40^{\circ}$ and increased significantly in the $40^{\circ}$ to $90^{\circ}$. And the lowest pressure value of the scapula and the hip-sacrum occurred in the $30^{\circ}$ and $40^{\circ}$ respectively. The largest pressure change curves of the shoulder blade and hip. $d_{1}$ is about $8 \mathrm{e} 3 \mathrm{~Pa}$ and $d_{2}$ is about $5.9 \mathrm{e} 4 \mathrm{~Pa}$. This is consistent with the previous finite element analysis results, which verifies the correctness of the biomechanical modeling.

\section{Discussion}


prone to occur in skeletal protuberance. The scapula and hip-sacrum are the most prominent bones which have a large area of action on human soft tissue, and most of the body weight concentrates in the upper body, so they can be considered the typical and main sites of pressure ulcers in the process of turning over from supine position. In order to estimate the biomechanical changes on the typical sites of pressure ulcers in the process of turning over, firstly, aimed at the biomechanical research of turning over from the supine position, the pressure distribution of the bone around the trunk's main sites of pressure ulcers was analyzed and theoretical contact pressure between the bone and the bed was calculated. Based on

Biomechanical model, the maximum stress change of those bones in different constraint conditions was simulated. Finally, the turning over experiment was carried on and the actual contact pressure between the typical sites of pressure ulcers and the bed was obtained. Theoretical calculation and simulation cannot simulate the real situation, and the experiment is also affected by many factors. But integration of theoretical analysis, experimental and simulation results, their trend and the numerical ratio of the calculation, simulation and the experiment are basically the same, and the study demonstrated the pressure changes of the trunk's typical sites of the pressure ulcers in the process of the turning over [32]. The finite element analysis results and the experiment results show that the maximum stress of the scapula and the hip-sacrum and the contact pressure of the shoulder-blade and hip are the same trend, and there is an angle threshold in these results that the contact pressure is smallest. An assisted exoskeleton robot designed for this angle threshold can keep the minimum pressure when the body is in contact with the bed. At the same time, the changes of the body's center of gravity are also known by the contact pressure cloud diagram, and it can provide a design principle 
that how to control the body's center of gravity and the assisted force. Relevant findings could be applied to pressure ulcer care, as well as assisted robotic control that follows the same trajectory as the human body.

Since the internal pressure of bone is not considered in the theoretical calculation and the boundary conditions set by finite element simulation are too ideal and too few, the theoretical calculation results and experiment results are certainly limited. In the following study, it will be further studied that the biomechanics of human bones in the process of turning over and to try to simulate the stress of bones in real situations.

\section{Conclusion}

In view of the current studies about compression of the typical sites of pressure ulcers are less, but pressure ulcer care is particularly important, so its biomechanics study is conducted, and the biomechanical changes on the typical sites of pressure ulcers in the process of turning over from supine position are revealed based on theoretical analysis, simulation and experiment. Among them, the complex skeletal movement was simplified into a staged mechanical model under the characteristic state to describe the contact between the typical sites of pressure ulcers and the bed surface during the whole rollover process, and the relationship between the contact pressure and angle is obtained. The results of calculation, simulation and experiment show the following rules: with the increase of the angle of turning over, the pressure on the shoulder increased gradually and the pressure on the hip decreased first and then increased. There is a phenomenon that the two lowest values appeared in the process of turning over and the largest value appeared in the position of $90^{\circ}$, and it suggests that turning over can relieve the high contact pressure between the body and the bed surface. At the same time, the largest pressure value occurs in the 
position of $90^{\circ}$ shows that the body position in a large angle of turning over should not be kept for long time. There may be an optimal angle for pressure ulcer care based on the smallest contact pressure of the typical sites. Decompression devices helpful to provide a reference for clinical nursing.

\section{Abbreviations}

CT: Computed tomography; MIMICS: Materialise's interactive medical image control system; CAD: Computer Aided Design.

\section{Acknowledgements}

The author wishes to thank National Natural Science Foundation of China (Grant No. 52005045), and National Key R\&D Program of China (Grant No. 2019YFC0119200), and Natural Science Foundation of Beijing Municipality (Grant No. 19L2018) that supported this work.

\section{Funding}

This study was funded by National Natural Science Foundation of China (Grant No. 52005045), and National Key R\& D Program of China (Grant No. 2019YFC0119200), and Natural Science Foundation of Beijing Municipality (Grant No. 19L2018).

\section{Availability of data and materials}

Data are available from the corresponding author upon reasonable request.

\section{Ethics approval and consent to participate}


The study was performed following the principles outlined in the Helsinki Declaration and it was approved by Clinical Research Ethics Committee. all the participants gave their informed consent.

\section{Conflict of Interests}

There are no conflicts of interest in this study.

Consent for publication

Consent for publication of individual data has been obtained from all the participants of the study.

\section{Authors' contributions}

All authors have made substantial contribution to this paper. among them, Peng Su and Leiyu Zhang firstly put forward to the conception of the study and QingLong Lun drafted the article then Peng Su revised it critically for intellectual content. And

Qinglong Lun, Da Lu, Tian Liu and QiuLong Wu were responsible to conduct experiment and analyze experiment date. Lastly, all authors read and approved the final manuscript.

\section{Author details}

${ }^{1}$ School of Electromechanical Engineering, Beijing Information Science and

\section{Reference}

[1] Jka B, Jc C, Kc D, Kb E, Dan B, H SL. Prevention and treatment of pressure 
ulcers/injuries: The protocol for the second update of the international Clinical Practice Guideline 2019. J Tissue Viability. 2019;28(2):51-58.

[2] Bhattacharya S, Mishra RK. Pressure ulcers: Current understanding and newer

[4] Yusuf S, Okuwa M, Shigeta Y. Microclimate and development of pressure ulcers and superficial skin changes. Int Wound J. 2015;12(1):40-46.

[5] Zhong W, Malcolm MQ, Q Xing, Pan N, Textiles and Human Skin, Microclimate, Cutaneous Reactions: An Overview. J Toxicol-Cutan Ocul. 2006;25(1):23-39.

[6] Dan L B, Worsley P R. Technologies to monitor the health of loaded skin tissues. BioMedical Engineering OnLine. 2018;17(1):40.

[7] Brem H, Maggi J, Nierman D. High cost of stage IV pressure ulcers. Am J Surg. 2010;200(4):0-477.

[8] Mc Ginnis, Briggs M, Collinson M. Pressure ulcer related pain in community populations: a prevalence survey. BMC Nurs. 2014;13(1):16.

[9] Kumari S, Sharma D, Rana A, Pathak R, Lal R, Kumar A. Risk assessment tool forpressure ulcer development in Indian surgical wards. Indian J Surg. 2015;77(3):206-212.

[10] Romanelli M, Clark M, Gefen A, Science and Practice of Pressure Ulcer Management. London: Springer; 2018.

[11] Wound, Ostomy and Continence Nurses Society-Wound Guidelines Task Force. WOCN 2016 Guideline for Prevention and Management of Pressure Injuries (Ulcers). Journal of WOCN Official Publication of the Wound Ostomy \& 
[12] Pickham D, Ballew B, Ebong K, Shinn J, Lough ME, Mayer B. Evaluating optimal patient-turning procedures for reducing hospital-acquired pressure ulcers (LS-HAPU): study protocol for a randomized controlled trial. Trials. 2016;17(1):190.

[13] Ning L, Peng Y, Yang T, Liang Z, Liu L, Bio-inspired wearable soft upper-limb exoskeleton robot for stroke survivors. 2017 IEEE International Conference on Robotics and Biomimetics (ROBIO). IEEE. 2017.

[14] Seo KH, Choi TY, Oh C. Development of a robotic system for the bed-ridden. Mechatronics. 2011;21(1):227-238.

[15] Mohammad, Karimi, Timon, Rabczuk, Mauludin, Luthfi. An evaluation of the efficiency of endpoint control on the correction of scoliotic curve with brace. A case study. Acta Bioeng Biomech. 2019;21(2):3-10.

[16] Derler S, Rao A, Ballistreri P, Huber R, Scheel-Sailer A, \& Rossi R M. Medical textiles with low friction for decubitus prevention. Tribology International. 2012;46(1):208-214.

[17] Rotaru GM, Pille D, Lehmeier FK. Friction between human skin and medical textiles for decubitus prevention. Tribology International. 2013;65:91-96.

[18] V Luís, R Amílcar, Friction of Human Skin against Different Fabrics for Medical Use. Lubricants. 2016;4(1):6.

[19] Defloor T. The effect of position and mattress on interface pressure. Applied Nursing Research Anr. 2000;13(1):2-11.

[20] Oomens CWJ, Broek M, Hemmes B. How does lateral tilting affect the internal strains in the sacral region of bed ridden patients? - A contribution to pressure ulcer prevention. Clin Biomech. 2016;35:7-13. 
440 [21] Sulagna, Sarkar, Tikeshwar, Prasad, Sahu, Arijit, Datta, Nimesh, Chandra.

441 Arindam Mechanical response at peri-implant mandibular bone for variation of

442 pore characteristics of implants: A Finite Element Study. Acta Bioeng Biomech.

$443 \quad 2019 ; 21(2): 83-93$.

444 [22] Kruszewski A, Piszczatowski S, Piekarczyk P, Kwiatkowski K. Evaluation of

445 stabilization of intra-articular fracture of distal humerus - finite element study.

$446 \quad$ Acta Bioeng Biomech. 2020;22(1):153-163.

447 [23] Ingram D. Musculoskeletal Model of the Human Shoulder for Joint Force

$448 \quad$ Estimation. Epfl. 2015.

449 [24] Shu J, Wang Q, H Ma, H Teng, Liu Z. Biomechanical study on the changes of

450 stress in temporomandibular joints after the orthognathic surgery in patients with

451 mandibular prognathism: a 3D finite element study. Acta Bioeng. Biomech.

$452 \quad 2020 ; 22(2): 155-163$.

453

454

455

456

457

458

459

460

461

462

463

464

[25] Shih-Chieh, Yang, Pao-Hsin, Liu, Yuan-Kun. Investigation of pullout strength in different designs of pedicle screws for osteoporotic bone quality using finite element analysis. Acta Bioeng Biomech. 2019;21(3):57-66.

[26] Schnackenburg K E, Macdonald H M, Ferber R, Wiley J P, Boyd S K. Bone quality and muscle strength in female athletes with lower limb stress fractures. Med Sci Sprots Exerc. 2011;43(11):2110-2119.

[27] Boyle C, Kim IY. Comparison of different hip prosthesis shapes considering micro-level bone remodeling and stress-shielding criteria using three-dimensional design space topology optimization. J Biomech. 2011;44(9):1722-1728.

[28] Martelli S, Taddei F, Cristofolini L. Extensive risk analysis of mechanical failure for an epiphyseal hip prosthesis: a combined numerical-experimental approach. 
Proc Inst Mech Eng H. 2011;225(2):126-140.

[29] Ahir SP, Walker PS, Squire-Taylor CJ, Blunn GW, Bayley J. Analysis of glenoid fixation for a reversed anatomy fixed-fulcrum shoulder replacement. J Biomech. 2004;37(11): 1699-1708.

[30] Polikeit A, Nolte LP, Ferguson SJ. The effect of cement augmentation on the load transfer in an osteoporotic functional spinal unit: finite-element analysis. Spine (Phila Pa 1976). 2003;28(10):991-996.

[31] Zhang L, Zhu M, Shen L, Liu Z. Finite element analysis of the contact interface between trans-femoral stump and prosthetic socket. Conference of the IEEE Engineering in Medicine and Biology Society. 2013:1270-1273.

[32] Nakhli Z, Hatira B, Pithioux M, Chabrand, P, Saanouni K, On prediction of the compressive strength and failure patterns of human vertebrae using a quasi-brittle continuum damage finite element model. Acta Bioeng Biomech. $2019 ; 21(2)$.

\section{Legends}

Fig.1 The typical sites of pressure ulcers in turning over from supine position.

Fig.2 Theoretical force analysis of the scapula in the process of turning over from supine position.

Fig.3 Theoretical force analysis of the hip-sacrum in the process of turning over from supine position.

Fig.4 Details of the model of the scapula and hip-sacrum.

Fig.5 The measurement of the contact pressure distribution.

Fig.6 The theoretical contact pressure of the scapula and hip-sacrum and the bed in different angle.

Fig.7 The finite element analysis outcome of the soft tissue of scapula and 
490 hip-sacrum.

491 Fig.8 The pressure distribution changes of scapula and hip in turning over from supine 492 position. 
Figures

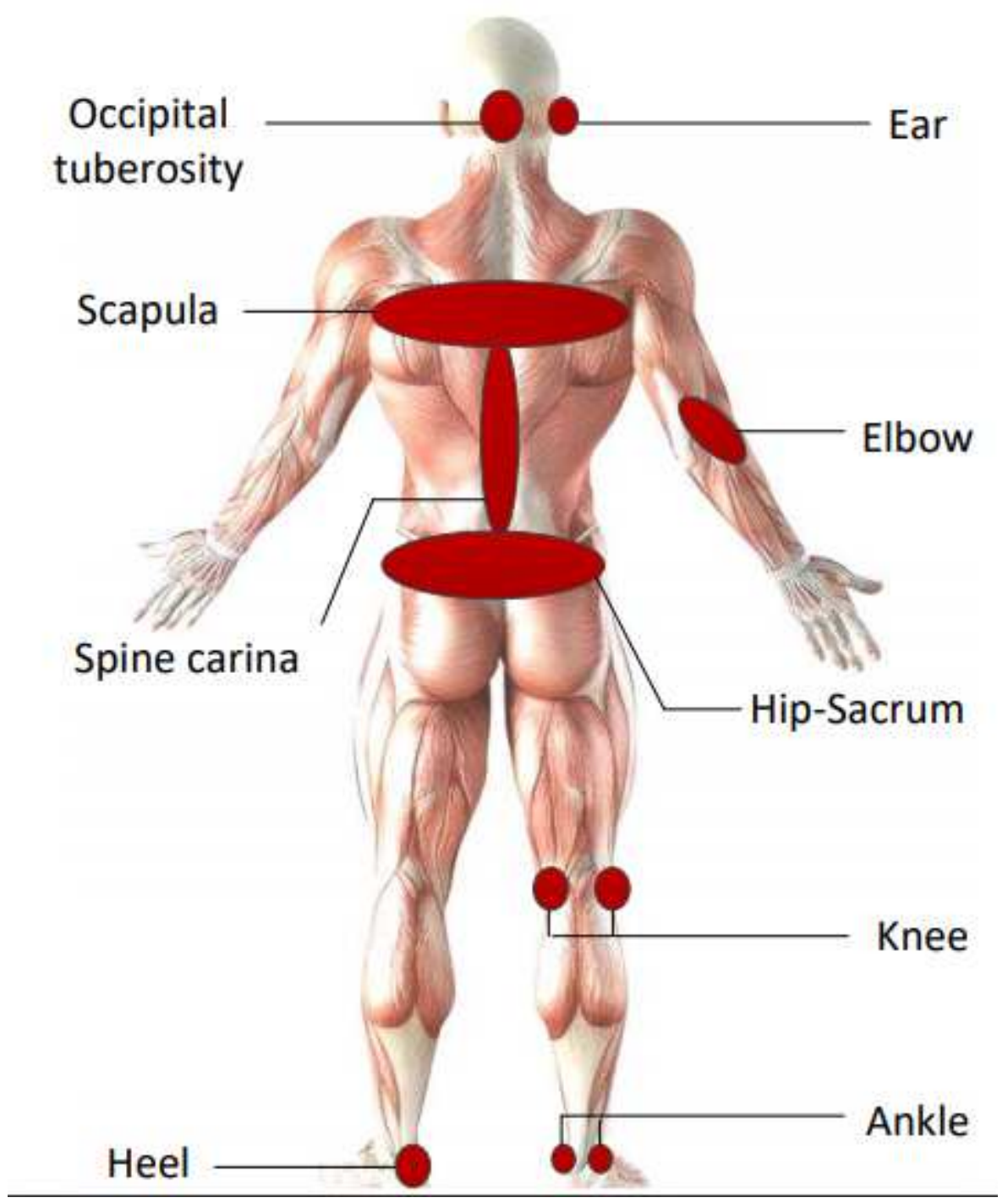

Figure 1

The typical sites of pressure ulcers in turning over from supine position. 


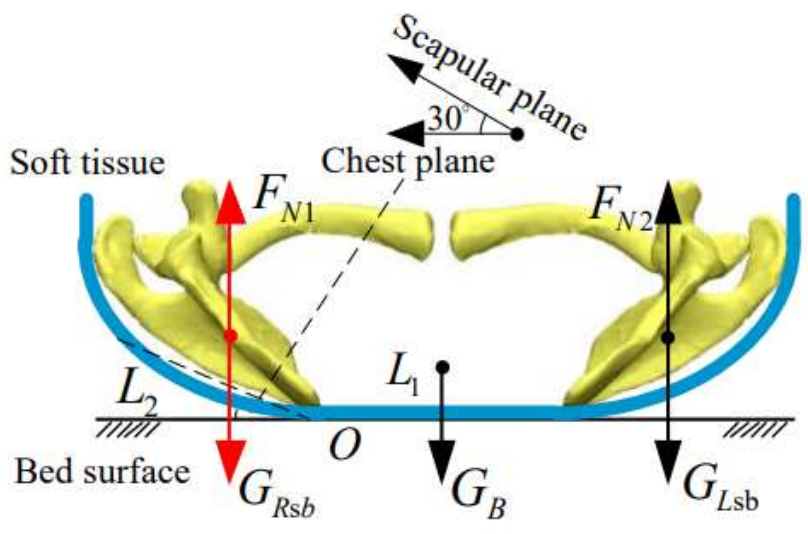

(a)

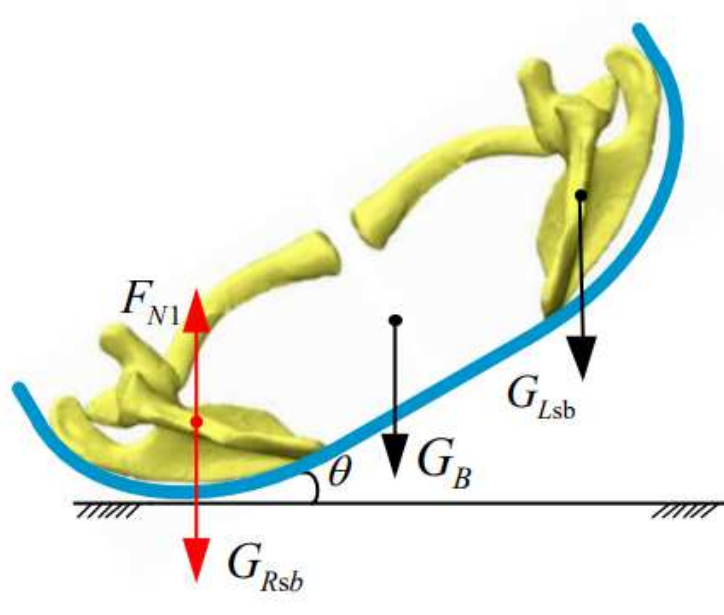

(b)

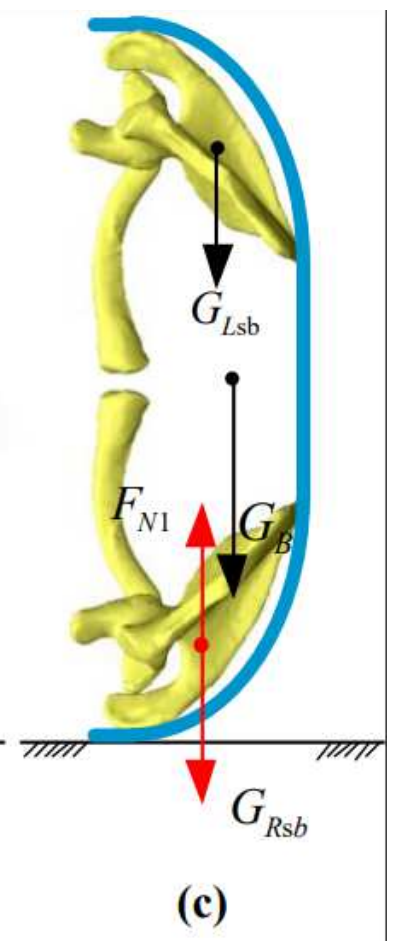

Figure 2

Theoretical force analysis of the scapula in the process of turning over from supine position.

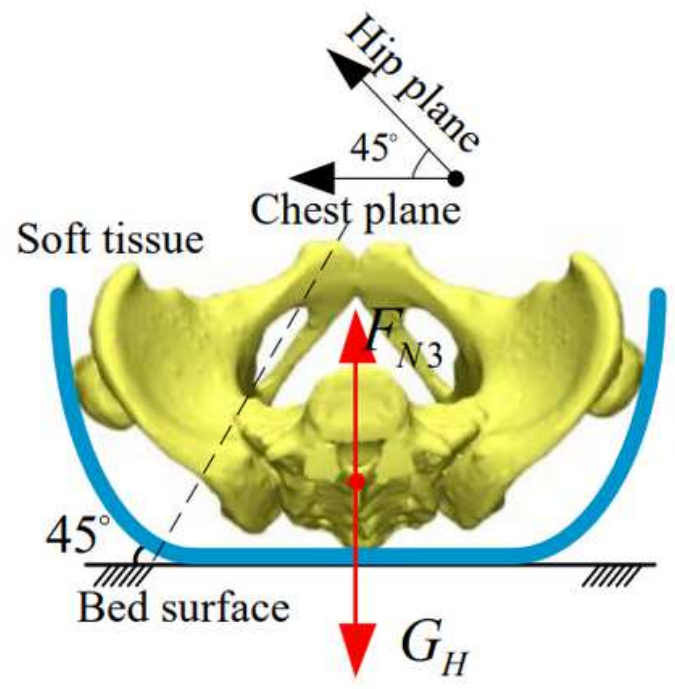

(a)

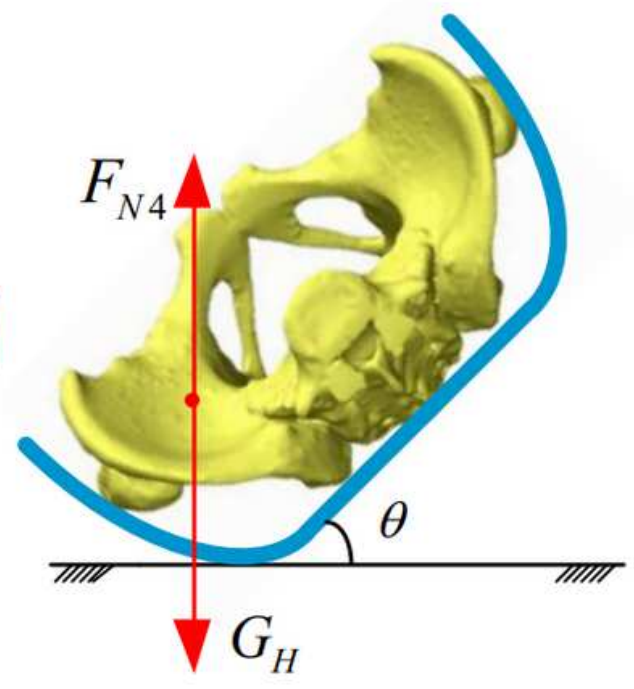

(b)

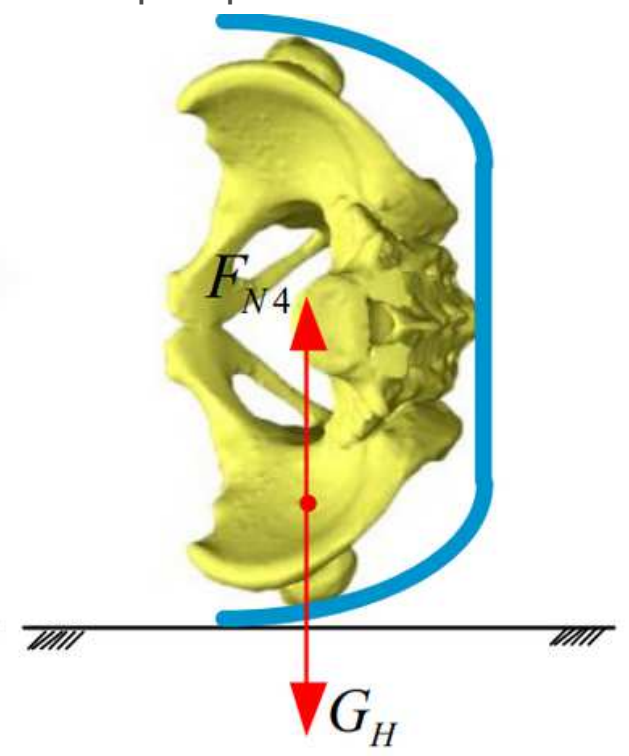

(c)

\section{Figure 3}

Theoretical force analysis of the hip-sacrum in the process of turning over from supine position. 
(1)

(2)

(3)

(4)

(8)

(9)

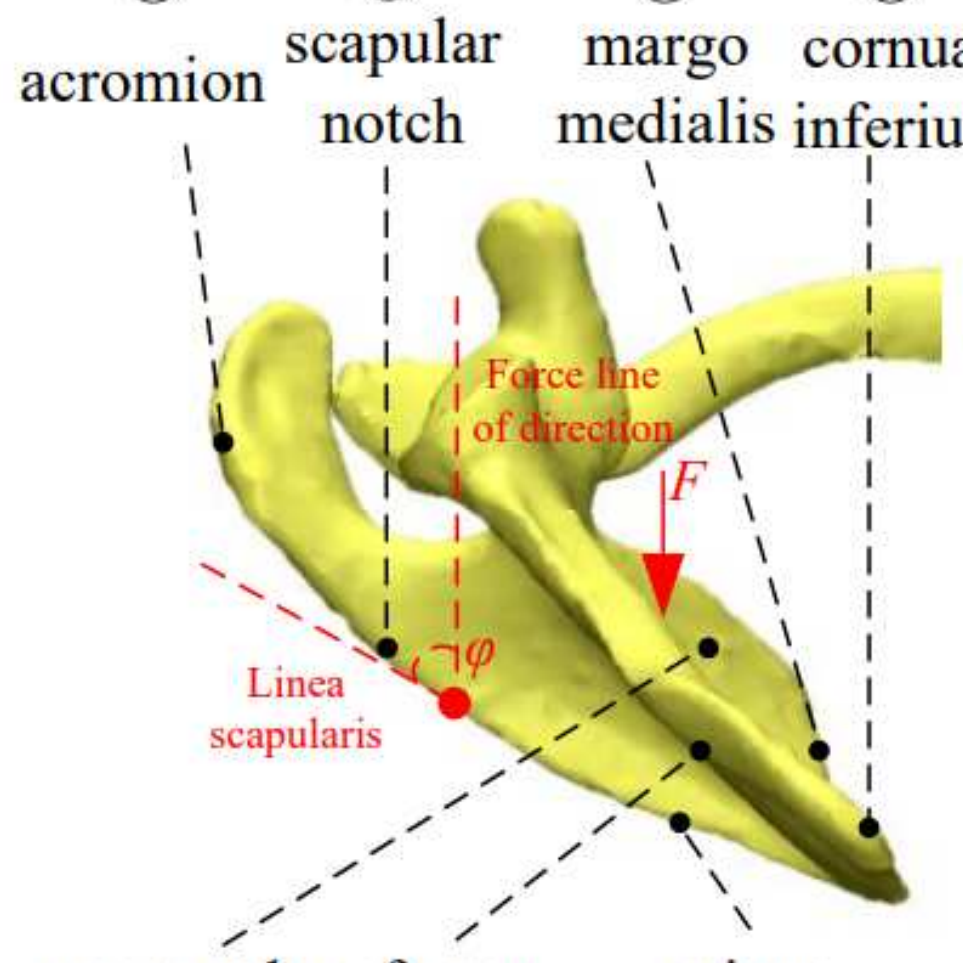

prescapular fossae spinae

fossae infraspinata scapulae

(5)

(6)

(a)
(7)

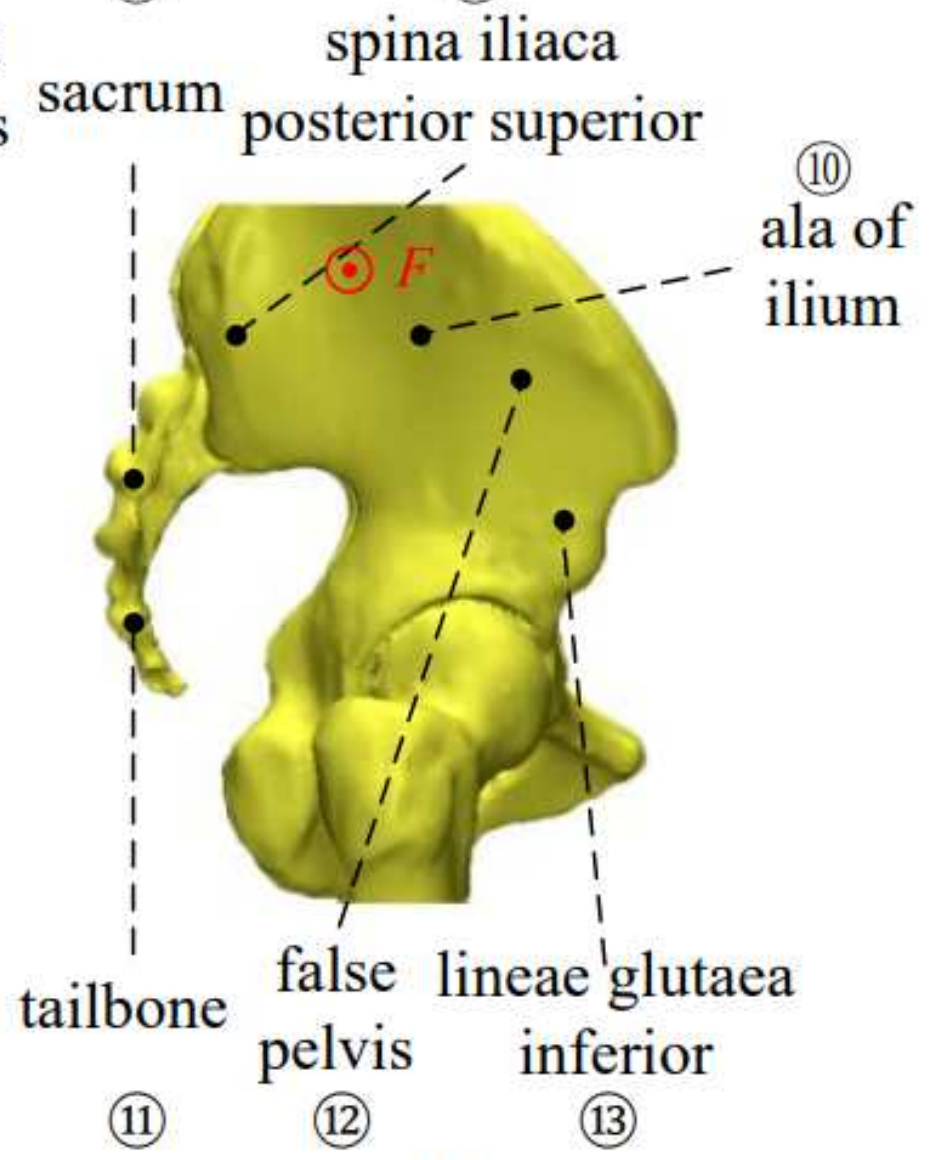

(b)

Figure 4

Details of the model of the scapula and hip-sacrum. 


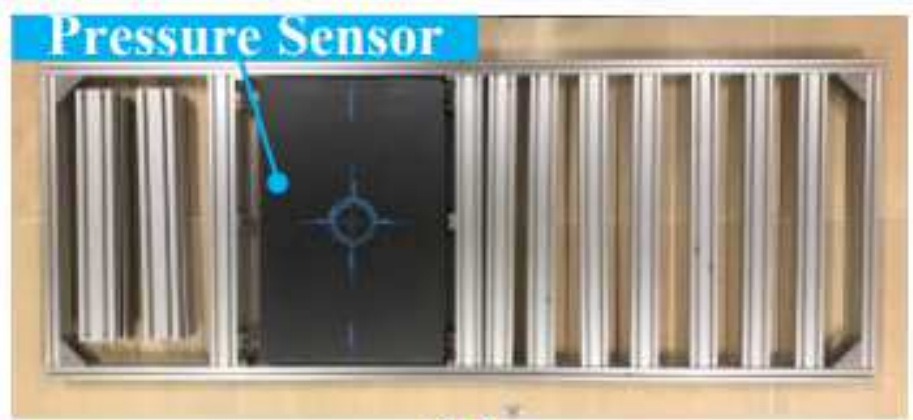

(a)

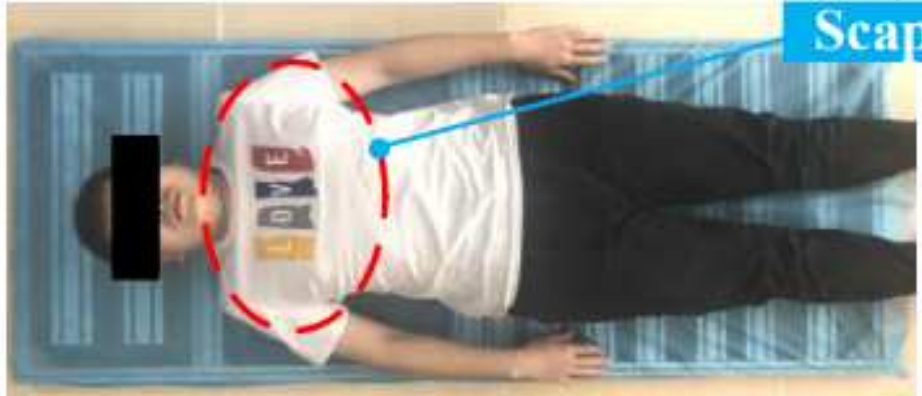

(b)

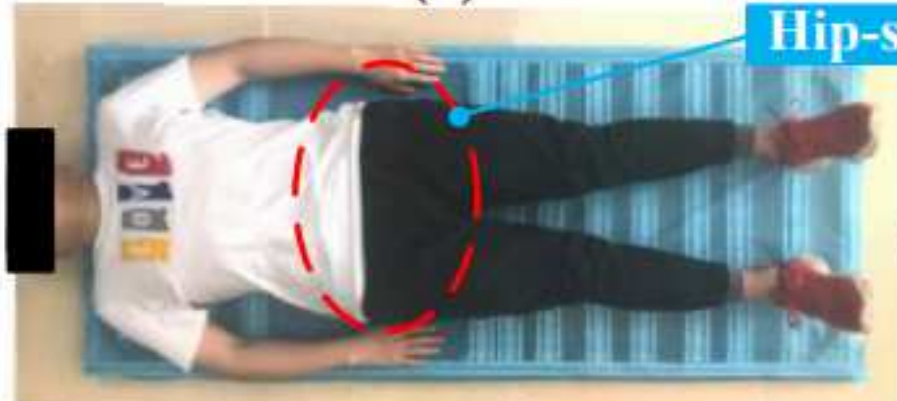

(d)

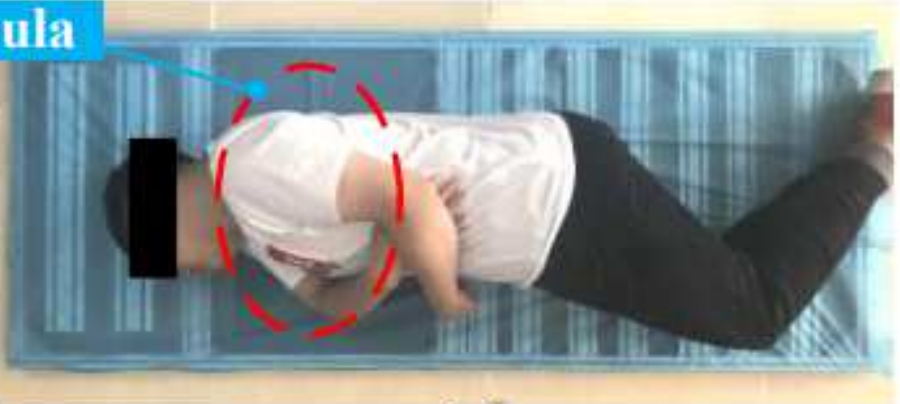

(c)

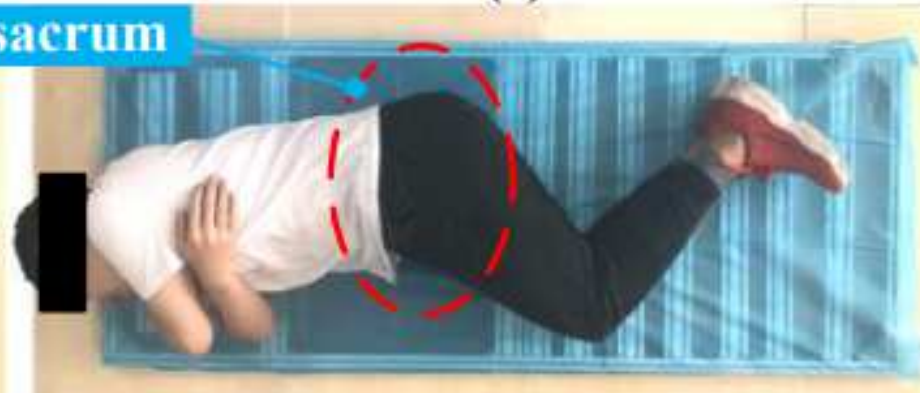

(e)

Figure 5

The measurement of the contact pressure distribution. 


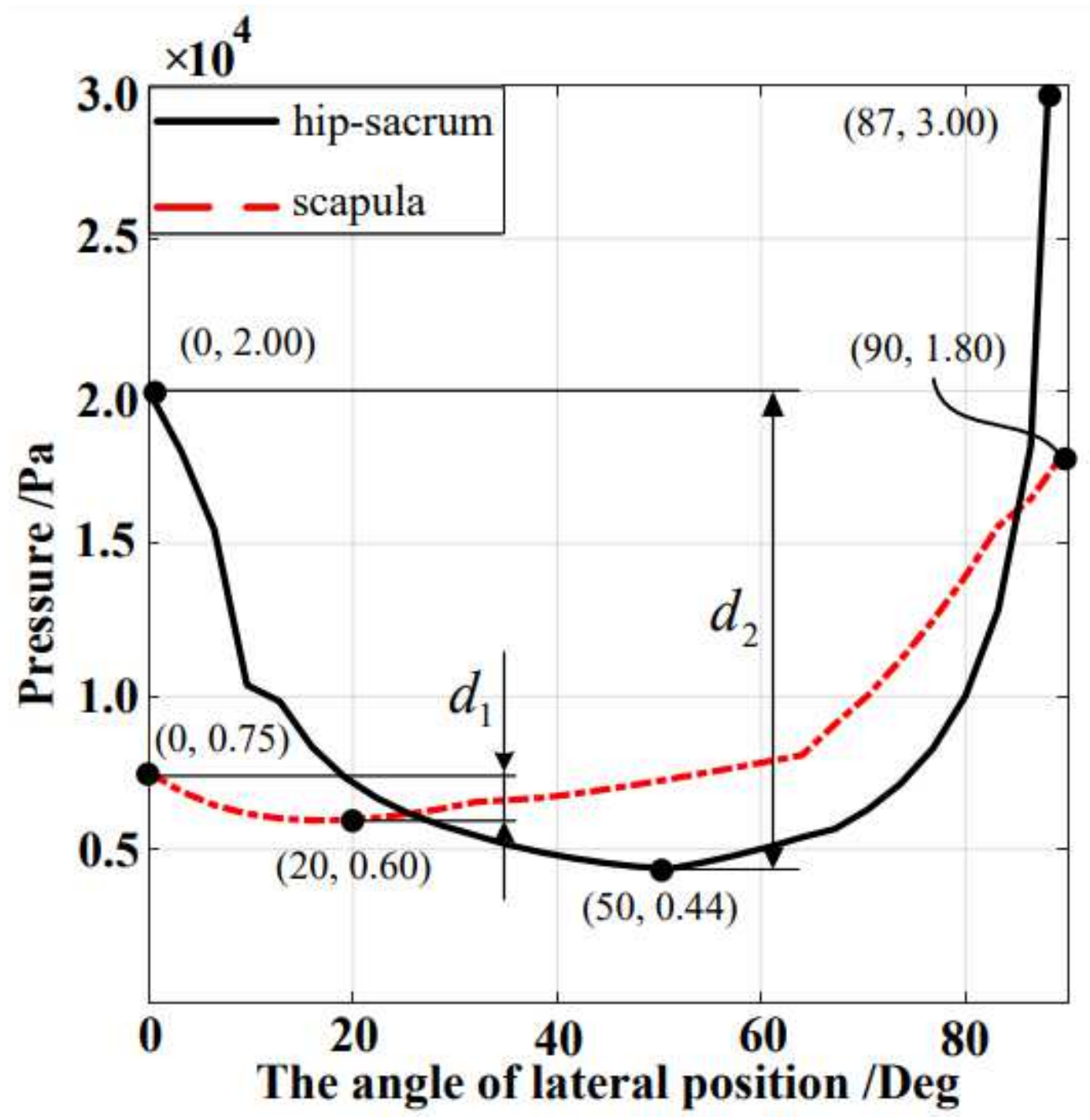

Figure 6

The theoretical contact pressure of the scapula and hip-sacrum and the bed in different angle. 


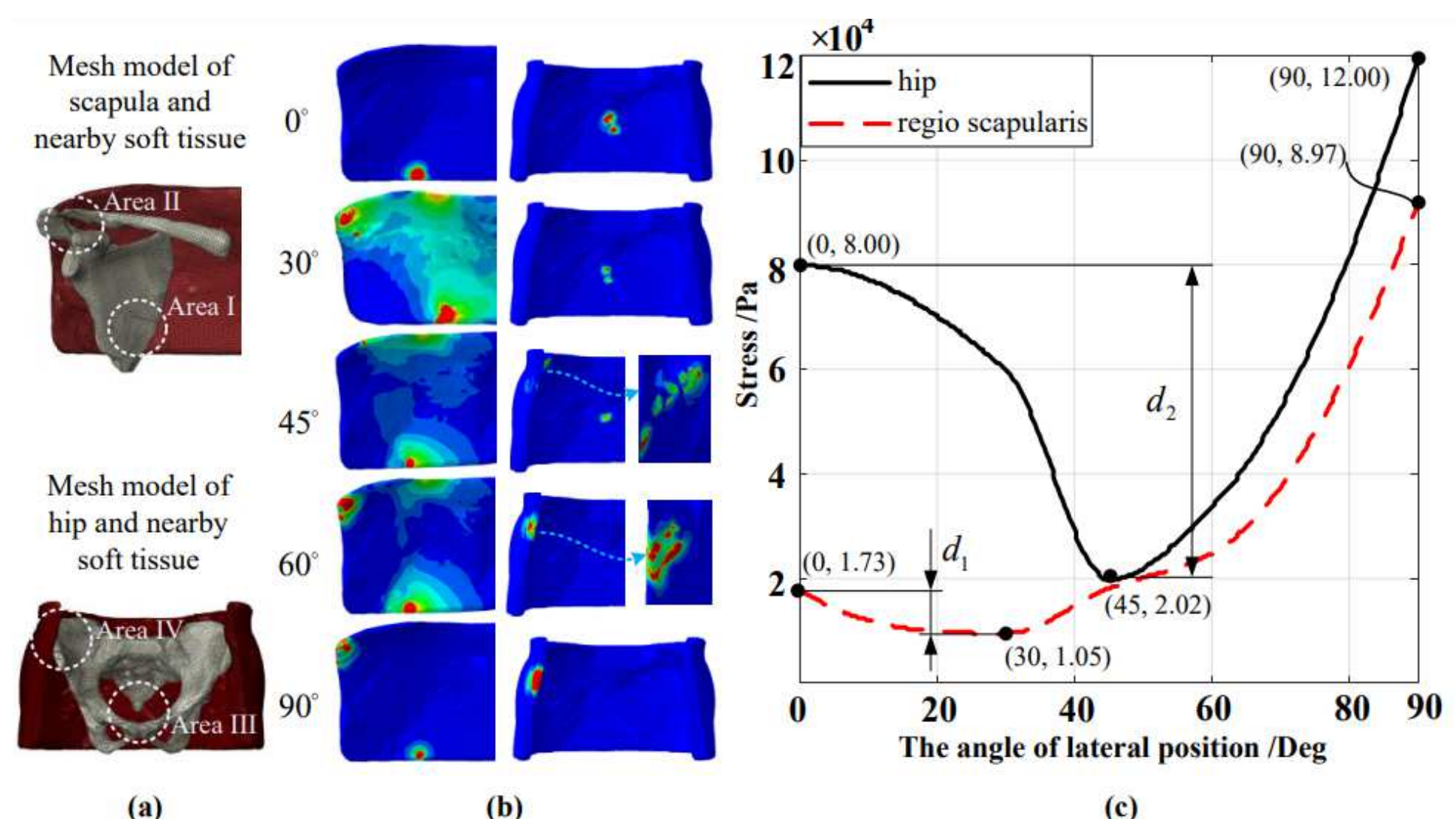

Figure 7

The finite element analysis outcome of the soft tissue of scapula and hip-sacrum. 


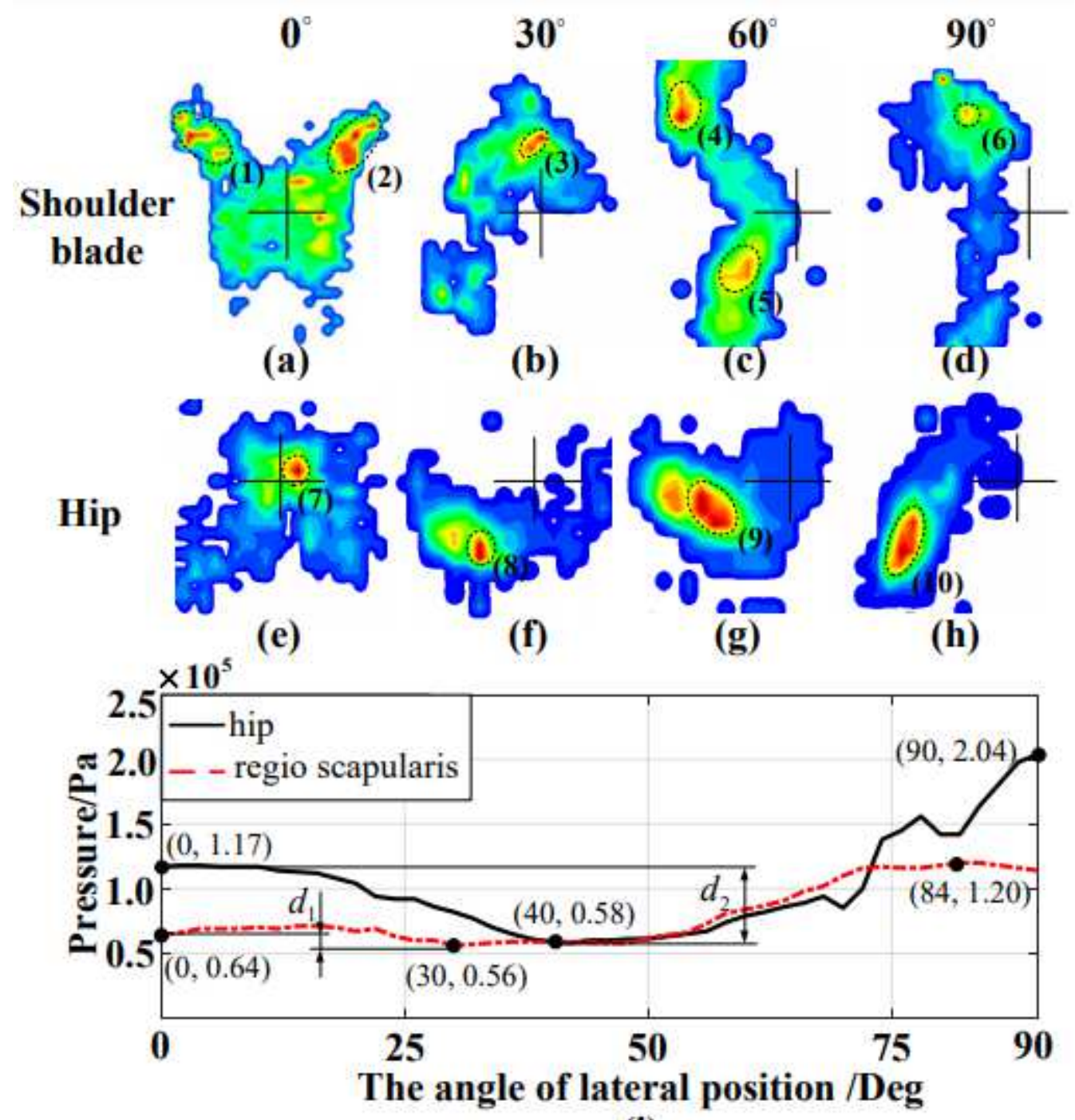

(i)

Figure 8

The pressure distribution changes of scapula and hip in turning over from supine position. 